\title{
Solid-solid transformations in a confined soft sphere fluid
}

\author{
Chandana Ghatak and K. G. Ayappa \\ Department of Chemical Engineering, Indian Institute of Science, Bangalore 560012, India
}

\begin{abstract}
Grand canonical Monte Carlo simulations are used to investigate the structure of frozen phases in a confined soft sphere (Lennard-Jones) fluid for a bulk state point lying close to the liquid-solid freezing line. The study reveals that the pore fluid not only freezes in the pore, but solid-solid transformations occur in the two, and three-layered structures that form as the pore height is varied. Using the in-plane bond angle order parameters and pair-correlation functions, the frozen structures and transformations are related to the variations in solvation force and pore density. Transformations from square to triangular lattices are observed in both the twoand three-layered regimes. In the three-layered regime, transformations occur from the body-centered tetragonal to hcp and fcc lattices as the pore height is increased. During the transition from one to two layers, random buckled structures are observed. The structural transformations from square to triangular lattices in the confined fluid also result in a splitting in the solvation force curve.
\end{abstract}

Confinement tends to reduce the dimensionality of the fluid giving rise to quasi-two-dimensional (2D) and quasi-3D systems. The physics of phase transformations in these lower-dimensional systems has attracted considerable interest, with particular emphasis on the 2D liquid to solid transition [1]. Reduced-dimensional systems occur naturally in a number of technologically important processes, such as adsorption, wetting, and lubrication. Using a combination of molecular simulations and density-functional theory, layering, gas-liquid, and liquid-solid transitions of molecularly confined fluids have been investigated [2], and with the exception of the liquid-solid or freezing transition, the thermodynamics of confined inhomogeneous fluids is well developed. Theoretical investigations of inhomogeneous fluids [3] at the molecular scale have been widely investigated using the prototype slit pore, which consists of two parallel walls. This model pore not only provides a convenient means of systematically varying the degree of confinement but also mimics the confinement geometry of the surface force apparatus [4] where forces exerted by molecular films are experimentally probed.

The surface force apparatus reveals the presence of oscillations in the normal force exerted by the fluid confined between atomically smooth mica surfaces. It is now well established that these oscillations are due to the formation and disruption of layers as the degree of confinement is changed [5]. The presence of a critical yield stress in dynamic surface force experiments of nonpolar organic molecules indicate solidification due to increased confinement leading to the classical stick-slip behavior [6].

Although the presence of substrate-induced or epitaxial freezing within confined fluid layers is well established [7], studies investigating liquid-solid or solid-solid phase transitions are few. Using a Landau free-energy approach, freezing of Lennard-Jones (LJ) fluids has been studied in smooth slit pores using grand canonical Monte Carlo (GCMC) simulations [8]. The simulations suggest that the liquid-solid (fcc lattice) transition occurs via an intermediate hexatic phase for strongly attractive walls. Monte Carlo studies of freezing in smooth slit pores show that the shift in freezing tempera- ture relative to the bulk is dependent on the nature of the walls $[9,10]$. GCMC simulations of LJ fluids confined between structured slit pores shows the presence of a solidsolid transition between square and triangular bilayer films [11]. These transitions, which were observed for incommensurate systems where the fluid molecular diameter was greater than the wall particle diameter, were not observed for the commensurate system.

Unlike molecularly confined fluids, phase transformations in confined colloidal suspensions naturally lends itself to direct optical imaging techniques. The cascade of solid-solid transitions between triangular and square lattices as the number of confined colloidal layers is increased is well established [12]. Monte Carlo simulations of hard spheres between hard walls in the single, and two-layer regimes, show that in addition to the solid-solid [13] transitions occurring between square to triangular lattices, buckled, and rhombic phases are also observed. Apart from the weak interaction with the walls, the similarities in the nature of confinement for colloidal systems, suggest that these transitions might occur in confined soft sphere fluids as well [11].

The aim of this paper is to investigate the structure of frozen phases and the transformation therein of an LJ fluid confined in a slit pore. We carry out an experimentally realizable situation using GCMC simulations, wherein the pore of fixed volume is equilibrated with a bulk fluid at fixed chemical potential and temperature. Unlike previous GCMC simulations, we consider a bulk state point that lies close to the liquid-solid freezing line. The proximity of the bulk liquid state point to the liquid-solid coexistence curve not only results in freezing of the confined fluid, but reveals the presence of solid-solid transitions as the pore height is varied to accommodate one, two, and three layers. We track the transitions by evaluating pair-correlation functions, bond angle order parameters, and correlate the structural changes with oscillations in the solvation force curve. We also make a qualitative comparison between the frozen phases observed in the soft sphere system investigated here with the solidsolid transitions observed in confined colloidal suspensions. 
The fluid-wall interaction $\left(U_{\mathrm{fw}}\right)$ is modeled using a 104-3 potential [15] with parameters similar to those used in previous simulations of methane on graphite [10],

$$
\begin{aligned}
U_{\mathrm{wf}}^{*}(z)= & 2 \pi \rho_{w}^{*} \epsilon_{\mathrm{wf}}^{*} \sigma_{\mathrm{wf}}^{* 2} \Delta^{*},\left[\frac{2}{5}\left(\frac{\sigma_{\mathrm{wf}}^{*}}{z^{*}}\right)^{10}-\left(\frac{\sigma_{\mathrm{wf}}^{*}}{z^{*}}\right)^{4}\right. \\
& \left.-\frac{\sigma_{\mathrm{wf}}^{* 4}}{3 \Delta^{*}\left(z^{*}+0.61 \Delta^{*}\right)^{3}}\right] .
\end{aligned}
$$

$z^{*}=z / \sigma_{\mathrm{ff}}\left(\sigma_{\mathrm{ff}}=3.81 \AA\right)$ is the perpendicular distance between the particle and the wall, $U_{\mathrm{wf}}^{*}=U_{\mathrm{wf}} / \epsilon_{\mathrm{ff}} \quad\left(\epsilon_{\mathrm{ff}}\right.$ $=148.1 \mathrm{~K}), \rho_{w}^{*}=\rho_{w} \sigma_{\mathrm{ff}}^{3}=6.305$ is the surface density of wall atoms, $\Delta^{*}=\Delta / \sigma_{\mathrm{ff}}=0.87926$ is the interlayer spacing of wall atoms, $\epsilon_{\mathrm{wf}}^{*}=\sqrt{\epsilon_{\mathrm{ww}} / \epsilon_{\mathrm{ff}}}=0.4348$ and $\sigma_{\mathrm{wf}}^{*}=0.5\left(1+\sigma_{\mathrm{ww}} / \sigma_{\mathrm{ff}}\right)$ $=0.9462$. All results are reported with respect to the reduced pore height, $H^{*}=H / \sigma_{\mathrm{ff}}$. Earlier studies for freezing in this system have shown that the structure of the frozen phase is not influenced by a more detailed fluid-wall potential describing the corrugation of the graphite sheet [10]. Fluidfluid interactions are modeled using a 12-6 LJ potential with $\epsilon_{\mathrm{ff}}$ and $\sigma_{\mathrm{ff}}$ as the two parameters. The bond angle order parameters

$$
\psi_{n}=\left\langle\frac{1}{N_{b}}\right| \sum_{j=1}^{N_{b}} \exp \left(\text { in } \theta_{j}\right)|\rangle,
$$

where $\theta_{j}$ is the bond angle formed between an atom and its nearest neighbors $\left(N_{b}\right)$ with reference to a fixed reference frame, are computed for for $n=4,6$. A distance cutoff of $1.25 \sigma_{\mathrm{ff}}$ was used while computing the nearest-neighbor bond distances for the bond order parameters. The in-plane paircorrelation functions, $g(r)$ are evaluated within each fluid layer. All bond angle order parameters reported here are averaged over the fluid layers in situations where more than one fluid layer forms in the pore. The solvation force $f_{z}^{*}$ $=f_{z} \sigma_{\mathrm{ff}}^{3} / \epsilon_{\mathrm{ff}}$ is the normal force per unit area exerted by the fluid molecules on each wall. The two, and three-layered structures observed here are classified based on their closest 3D counterparts. The in-plane lattice parameters $a^{*}=b^{*}$ were estimated from the peaks in $g(r)$ and $c^{*}$ was computed from the peak positions of the layered density distributions to within an accuracy of $0.02 \sigma_{\mathrm{ff}}$.

During the GCMC $(\mu \mathrm{VT})$ simulations, $4-5 \times 10^{6}$ Monte Carlo moves were used for equilibration, followed by $5-6 \times 10^{6}$ moves during which ensemble averages were collected. Longer runs, especially in the regions where the phase transitions were observed, did not alter the results. Each Monte Carlo move consists of an attempted addition, deletion, and displacement with equal probability [16]. The pore fluid is equilibrated with a bulk fluid whose activity, $Z^{*}=Z \sigma_{\mathrm{ff}}^{3}=1.2, T^{*}=k T / \epsilon_{\mathrm{ff}}=1.0$, where $k$ is the Boltzmann constant. This state point corresponds to a bulk liquid density, $\rho^{*}=\rho \sigma_{\mathrm{ff}}^{3}=0.898$, located close to the solid-liquid coexistence line [17]. A square simulation box was employed in all cases with periodic boundary conditions in the $x-y$ plane. The results reported here correspond to a periodic box length of $9 \sigma_{\mathrm{ff}}$ and a potential cutoff of $4.5 \sigma_{\mathrm{ff}}$.
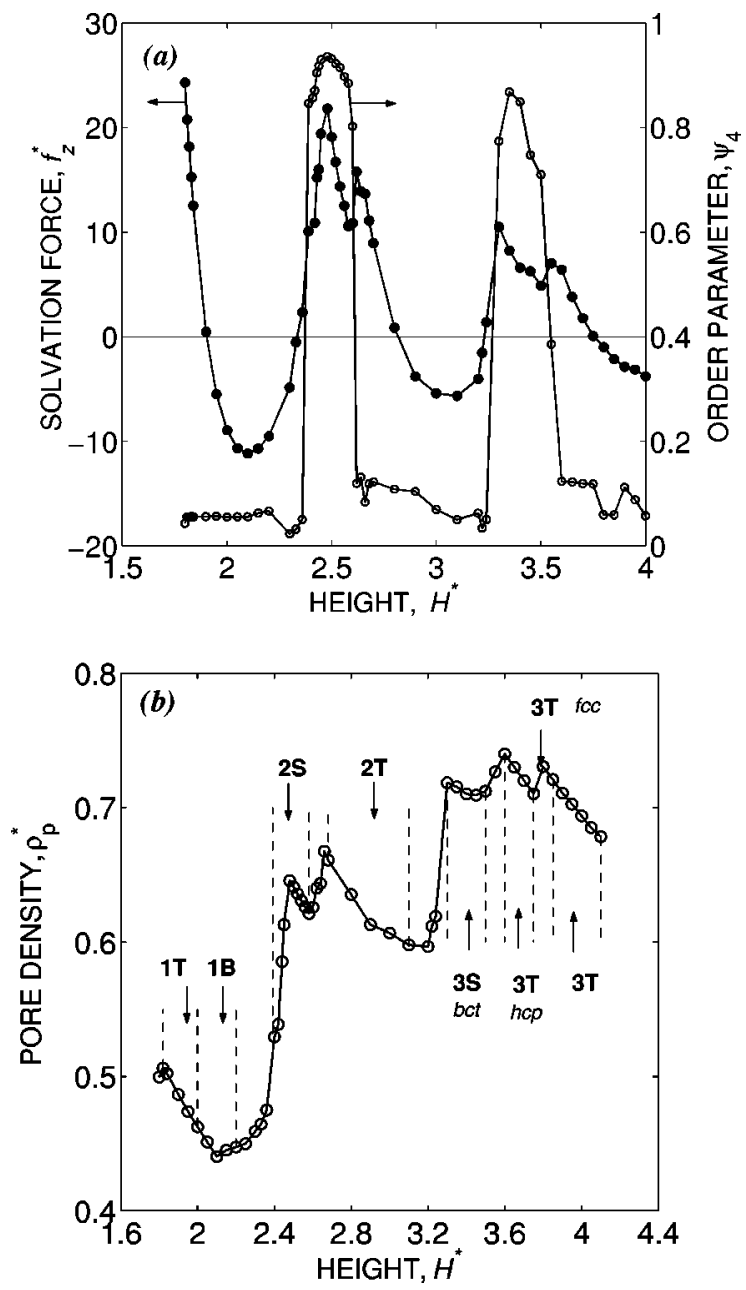

FIG. 1. (a) Reduced solvation force, $f_{z}^{*}=f_{z} \sigma_{\mathrm{ff}}^{3} / \epsilon_{\mathrm{ff}}$, which is the normal force per unit area exerted by the fluid molecules on the wall and bond angle order parameter $\psi_{4}$ as a function of the reduced slit height, $H^{*}=H / \sigma_{\mathrm{ff}}$. The splitting in the solvation force peaks, in the two- and three-layered regimes occurs during the transition between square to triangular lattices as seen by the rapid change in $\psi_{4}$ through this region. (b) Reduced pore density, $\rho_{p}^{*}$ $=\rho_{p} \sigma_{\mathrm{ff}}^{3}$ vs $H^{*}$ denoting the various phases observed. T-triangular, B-buckled, S-square with numerical prefixes indicating the number of layers. The regions where the structures are not marked indicate transitions between layers or phases. The three-layer fcc structure is observed at $H^{*}=3.8$.

Figure 1(a) shows the solvation force and bond angle order parameter $\psi_{4}$ as a function of the reduced slit height $H^{*}$. The maxima in the solvation force correspond to wellordered fluid layers and the minima correspond to regions where a transition between layers occurs. From the density distributions, the following regimes, within the resolution of slit heights $\left(\Delta H^{*}=0.03\right)$ used in our simulations, may be discerned; single layer $1.8 \leqslant H^{*} \leqslant 2.1$; transition between one and two layers, $2.1<H^{*} \leqslant 2.36$, two layers $2.39 \leqslant H^{*}$ $\leqslant 3.1$; transition between two and three layers $3.2 \leqslant H^{*}$ $<3.3$, and three layers $3.3 \leqslant H^{*} \leqslant 4.1$. The transition regimes where layering is disrupted, occurs in a small range of pore heights indicating that the confined fluid is in a highly layered state. The solvation force curve reveals the presence of 


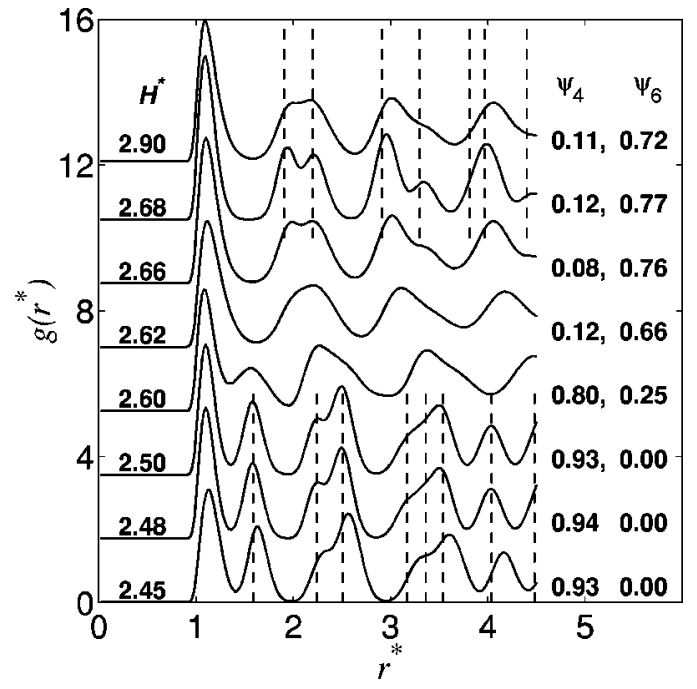

FIG. 2. In-plane pair-correlation functions for the two-layered regimes illustrating the transition from a square to triangular lattice. The vertical dashed lines indicate the location of the peaks for an ideal (zero-temperature) lattice. The lattice parameters were estimated from the location of the first peak of the pair-correlation functions. The bond order parameters are shown as well. $H^{*}$ $=2.62,2.66$ represent the transition regimes between square and triangular lattices.

split peaks in the two- and three-layered regimes. This splitting of peaks in the solvation force curve in the two-layer regime has been observed by Bordarier, Rousseau, and Fuchs [11] where a transition from a bcc to triclinic structure was observed for a fluid confined in a structured slit pore. We next discuss the structure of the pore fluid as inferred from the bond angle order parameters and the in-plane paircorrelation functions, focusing on the two- and three-layer regimes where phase transitions are observed, and relate the evolution of structures to the solvation force curve and pore fluid densities shown in Fig. 1(b).

The in-plane $g(r)$ 's for one of the wall layers in the twolayered regime are shown in Fig. 2. The vertical lines correspond to the peak positions for an ideal zero-temperature lattice based on the lattice parameter obtained from the first peak of the $g(r)$. The square lattice first forms at $H^{*}$ $=2.39,\left(\psi_{4}=0.84\right)$ where the pore fluid density shows a sharp increase [Fig. 1(b)]. The $g(r)$ in Fig. 2 is first shown at $H^{*}=2.45 \quad\left(\psi_{4}=0.93\right)$ where the peaks clearly reveal the presence of a square lattice. At $H^{*}=2.48\left(\psi_{4}=0.94\right)$ where the pore fluid density and solvation force achieves a local maximum, the order parameter is the largest value observed in our system. Beyond $H^{*}=2.48$, the pore fluid density decreases [Fig. 1(b)] with a concomitant lowering of the solvation force and increasing disorder till $H^{*}=2.58$. For 2.58 $<H^{*} \leqslant 2.66$, in-plane order increases and the fluid transforms to a triangular $(111 \mathrm{fcc})$ lattice at $H^{*}=2.68$ as seen in the corresponding $g(r)$ (Fig. 2). The transformation to a triangular lattice is accompanied by an increase in pore density and solvation force [Figs. 1(a) and 1(b)]. During the transformation, a splitting in the solvation force peak [Fig. 1(a)] is observed. The sharp drop in the order parameter $\left(\psi_{4}\right)$ in this region confirms that the minima in the solvation force occurs

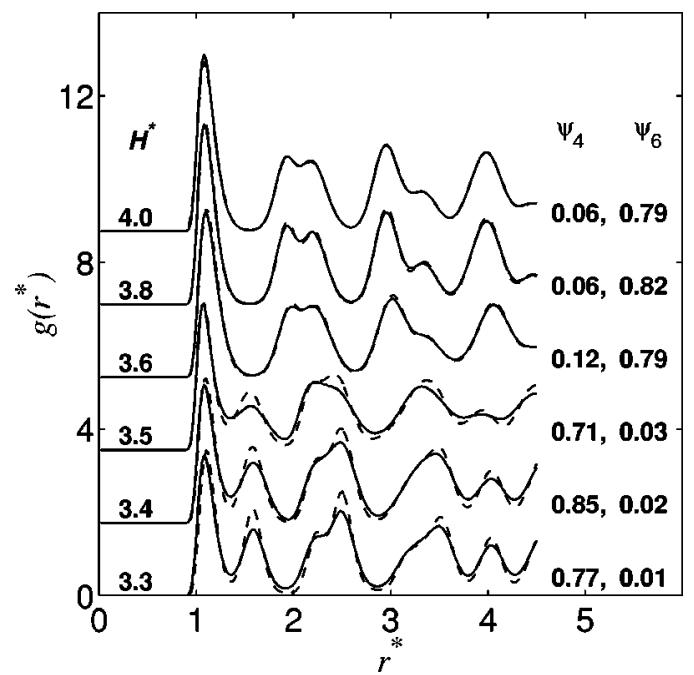

FIG. 3. In-plane pair-correlation functions for the three-layered regime. The pair correlation for the central layer (dashed lines) show greater in-plane order. In addition to the transition from square to triangular lattices, there exists a transition from the hcp $\left(3.6 \leqslant H^{*}<3.8\right)$ to the fcc structure at $H^{*}=3.8$. For $H^{*}$ $>3.8$, increased buckling in the central layer is observed.

during the transition between two lattice structures [Fig. 1(a)]. The triangular lattice structure persists till $H^{*}=2.9$ $\left(\psi_{6}=0.74\right)$, beyond which the fluid enters into a narrow transition regime between two and three layers. The lattice at $H^{*}=2.48$ corresponds to the first two basal planes of a body-centered tetragonal (bct) lattice, where $a^{*}=b^{*}$ $=1.12 ; c^{*} / 2=0.66$ and the structure at $H^{*}=2.68$ corresponds to the first two basal planes of the hcp structure, where $a^{*}=b^{*}=1.1 ; c^{*} / 2=0.86$.

In the three-layered situations, the structures are able to form a complete unit cell in the direction of confinement. The corresponding $g(r)$ plots for the three-layered system are shown in Fig. 3. The $g(r)$ 's for the contact layer and middle layer are shown for $3.3 \leqslant H^{*} \leqslant 4.0$. The presence of order in the contact layers induces greater order in the middle layer where the contact layer plays the role of a psuedostructured wall, into which the middle layer "epitaxially" orders. The square lattice exists for $3.3 \leqslant H^{*} \leqslant 3.5$. At $H^{*}=3.3$ where $a^{*}=b^{*}=1.09 ; c^{*}=1.48$, the structure corresponds to a unit cell of the bct lattice. At $3.5<H^{*}<3.55$, where the transition from a square to triangular lattice occurs, the $g(r)$ 's indicate that disorder first occurs in the wall layer. At $H^{*}=3.6$, the fluid transforms into a triangular lattice. This is accompanied by an increase in density [Fig. 1(b)]. Here, as in the case of the two-layered regime, the local minima in the solvation force observed at $H^{*}=3.5$ [Fig. 1(a)] occurs at the onset of the transition from square to a triangular lattice as indicated by the sharp drop in the order parameter $\psi_{4}$ [Fig. $1(\mathrm{a})]$ in this region.

In addition to the square-to-triangular lattice transition, an additional solid-solid transition was observed in the threelayered triangular lattice based on the stacking of individual layers. Examination of the stacking sequence in the threelayered lattices reveals an $A B A$ stacking for $3.6 \leqslant H^{*}<3.8$ corresponding to the hep lattice. At $H^{*}=3.8$, the stacking 
sequence transformed to the $A B C$ stacking of an fcc crystal. For $3.8<H^{*} \leqslant 4.1$, although the in-plane triangular lattice structure is preserved, the relative arrangement of atoms between layers deviates from the ideal lattice structures. Outof-plane displacements (buckling) in the central layer for $H^{*}>3.8$ increase sharply with deviations as large as $0.5 \sigma_{\mathrm{ff}}$ at $H^{*}=4.0$. Examinations of configurations in this region reveal that the atoms of the buckled central layer no longer occupy the threefold sites formed by the wall layer fcc lattice, but are shifted preferentially toward one of three atoms. This preferential shift was also observed in the two-layered regime for $2.9 \leqslant H^{*} \leqslant 3.1$, indicating that this relative shifting of atoms from their ideal locations could also be attributed to the increasing interplanar distance. The in-plane triangular structure persists until $H^{*}=4.1$ above which the transition into four layers occurs. At $H^{*}=3.6, a^{*}=b^{*}$ $=1.1 ; \quad c *=1.74, \quad$ and at $H^{*}=3.75, \quad a^{*}=b^{*}=1.1 ; \quad c *$ $=1.89$. These correspond to $c / a$ ratios of 1.58 and 1.72 , respectively. For the ideal hcp structure of spheres $c / a$ $=1.633$.

Before concluding, we briefly discuss the single-layer regime and contrast our paper with the hard-sphere phase diagram where the transition between single-layer triangle to two-layered square structures to proceed via buckled phases and the two-layer square to triangular transition occurs via an intermediate rhombic phase [13]. In the single-layer regime $\left(1.8 \leqslant H^{*} \leqslant 1.9\right)$ the fluid structure is that of an $111 \mathrm{fcc}$ lattice. In our system, buckling occurs in the region $2.0 \leqslant H^{*}$ $\leqslant 2.25$, where the order parameters and $g(r)$ indicate that the underlying lattice is triangular. However, buckling was neither zigzag or linear, as observed in the hard-sphere system, but random in nature. The rhombic phase, if present, would manifest between $2.58<H^{*}<2.62$, where the transition occurs from the two-layer square to triangular. However, we are inconclusive about the existence of this phase and perhaps more detailed simulations with finer resolution are probably required to isolate this phase. The prism phase [14] that was observed between the transition from triangular to square lattices for two-layered structures and above were not observed in our simulations. We point out that unlike the colloidal systems where the experiments probe the hardsphere packing limit in a continuous fashion, our simulations are carried out in an open system where the pore density is determined by the bulk reservoir conditions. The transitions between triangular and square lattices occur via an intermediate disordered phase corresponding to a minima in the solvation force response.

To summarize, we have shown that the proximity of the bulk state to the liquid-solid freezing line may induce solidsolid phase transitions in confined soft sphere systems that persist beyond the two-layered regime. The qualitative features of these transitions show a remarkable similarity to those observed in confined colloidal crystals and the various phases are summarized in Fig. 1(b). The transitions between square and triangular lattices in both the two- and threelayered systems are accompanied by a splitting in the solvation force peaks. Significant in our findings is the transitions between bct, hcp, and fcc lattices in the three-layered regimes. Our simulations suggest that the structural transitions in confined soft sphere systems is far richer and more complex than previously understood.

We are grateful to R. M. Lynden Bell and T. A. Abinandanan for several useful discussions related to this paper.
[1] Bond-Orientational Order in Condensed Matter Systems, edited by K. J. Strandburg (Springer-Verlag, New York, 1992).

[2] L. D. Gelb, K. E. Gubbins, R. Radhakrishnan, and M. Sliwinska-Bartkowiak, Rep. Prog. Phys. 62, 1573 (1999).

[3] H. T. Davis, Statistical Mechanics of Phases, Interfaces and Thin Films (VCH, Wienheim, 1993).

[4] J. Israelachvili, Intermolecular and Surface Forces (Academic, London, 1992).

[5] I. K. Snook and W. van Megen, J. Chem. Phys. 72, 2907 (1980); J. J. Magda, J. Tirrell, and H. T. Davis, ibid. 83, 1888 (1985); J. Gao, W. D. Luedtke, and U. Landman, Phys. Rev. Lett. 79, 705 (1997).

[6] M. L. Gee, P. M. McGuiggan, and J. N. Israelachvili, J. Chem. Phys. 93, 1895 (1990); S. Granick, Science 253, 1374 (1992); J. Klein and E. Kumacheva, J. Chem. Phys. 108, 6996 (1998); 108, 7010 (1998).

[7] M. Schoen, D. J. Diestler, and J. H. Cushman, J. Chem. Phys. 87, 5464 (1987); C. L. Rhykerd Jr., M. Schoen, D. J. Diestler, and J. H. Cushman, Nature (London) 330, 461 (1987); J. Gao, W. D. Luedtke, and U. Landman, J. Phys. Chem. B 101, 4013 (1997).
[8] R. Radhakrishnan and K. E. Gubbins, Mol. Phys. 96, 1249 (1999).

[9] H. Dominguez, M. P. Allen, and R. Evans, Mol. Phys. 96, 209 (1999).

[10] M. Miyahara and K. E. Gubbins, J. Chem. Phys. 106, 2865 (1997).

[11] P. Bordarier, B. Rousseau, and A. H. Fuchs, Mol. Simul. 17, 199 (1996).

[12] P. Pieranski, L. Strzelecki, and B. Pansu, Phys. Rev. Lett. 50, 900 (1983); D. H. Van Winkle and C. A. Murray, Phys. Rev. A 34, 562 (1986).

[13] M. Schmidt and H. Löwen, Phys. Rev. Lett. 76, 4552 (1996); Phys. Rev. E 55, 7228 (1997).

[14] S. Neser, C. Bechinger, and P. Leiderer, Phys. Rev. Lett. 79, 2348 (1997).

[15] W. A. Steele, Interaction of Gases with Solid Surfaces (Pergammon, Oxford, 1974).

[16] M. P. Allen and D. J. Tildesley, Computer Simulation of Liquids (Clarendon, Oxford, 1987).

[17] R. Agarwal and D. A. Kofke, Mol. Phys. 85, 43 (1995). 